\title{
INTER-ORGANIZATIONAL WORKFLOW MANAGEMENT IN VIRTUAL HEALTHCARE ENTERPRISES
}

\author{
Tauqir Amin, Pung Hung Keng \\ Department of Computer Science, National University of Singapore \\ [amin.punghk]@comp.nus.edu.sg \\ SINGAPORE
}

\begin{abstract}
Virtual enterprise provides an attractive model for autonomous healthcare organizations to synergize and leverage the strengths of each others. We present in this paper the design concept and working principle of an interorganizational workflow process management system for virtual healthcare enterprises. We propose an extended WFMC process Meta model and a new concept of Partial View of Virtual Process for representation and enactment of cross-organizational processes. Using this approach, local processes of participating organizations can be integrated with other external processes quickly. The joining and disjoining a virtual enterprise by an organization is also transparent to all except the directly interacting organizations.
\end{abstract}

\section{INTRODUCTION}

\subsection{Motivation}

In order to be competitive in the dynamic marketplace, healthcare enterprises need to deploy information technology solutions that internally automate the paper-based medical record systems and externally create smart connections between the major participants and collaborators in health care. With the emergence of near ubiquitous Internet connectivity, a virtual integrated health-care organization built upon connections, partnerships, alliances, and relationships with physicians, polyclinics, laboratories, pharmacies and hospitals is an emergent operating model for e-health organizations [1]. While the information systems of participating organizations are capable of handling internal administrative tasks and clinical processes [2], they are often not design with an objective of supporting collaborations with external organizations. The need for integrating the workflow processes of interorganizations is therefore essential for the realization of virtual healthcare enterprises.

\subsection{Problem statement}

The most relevant activities within a healthcare organization can be described in the form of business processes as mentioned in [3]. Following this idea, we define a 
virtual process as a business process whose definition and enactment is distributed among more than one healthcare organization. From here, we define virtual health enterprises as those whose business processes are virtual processes. The potential participating organizations may have their own internal processes that were developed previously without considering that they could be part of any virtual process. Therefore building and enacting a virtual process from such local processes is a big challenge. This challenge is intensified when dynamics of relationships, autonomous behavior of local processes and heterogeneity of workflow management systems (WfMSs) are considered. To address the challenge, changes in a process meta model and a process enactment framework are required. These changes should be in such a way that they can easily be adopted by systems based on the WfMS' standard.

\subsection{Overview}

To solve the above problem, we introduce a concept of "partial views". To an organization a "partial view of virtual process" is the part of whole virtual process seen by it. Such partial view of virtual process consists of organization's local process and parts of the processes of other interacting organizations. We developed Meta models for partial view of virtual processes and also top-level entities of the virtual process enactment framework. The framework is distributed and based on a collection of autonomous, physically apart, possibly heterogeneous workflow management systems connected in a loosely coupled way, most likely through the Internet. The hook-up procedure for making one virtual clinical or administrative process is bottom up. For process modeling, we extended modeling constructs of reference Meta model of process definition of the Workflow Management Coalition (WFMC) [4].

Section 2 presents Meta model of partial view of virtual process and in Section 3, after presenting an illustrative example, the applications of this approach are discussed. Section 4 describes the architecture and entities of the virtual process enactment framework. Through discussion of major pros and cons of the solution, an evaluation of the solution is given in Section 5. Related work and conclusions are presented in Section 6 and Section 7 respectively.

\section{META MODEL FOR PARTIAL VIEW OF VIRTUAL PROCESS}

Our Meta model for partial view of virtual process is actually an extension of the Meta model of the process definition as specified by WFMC [4]. We added five new notions, which are shown as shaded blocks in the figure 1. For the sake of simplicity of the figure, two standard types of activity (dummy, loop) are not shown.

The entity proxy process represents an external process and during enactment it would be realized by the external WfMS. Proxy process consists of proxy activities that represent exposed activities of the external process. In other words, proxy activity or proxy process substitutes some real activities or processes running on some other workflow management systems. From the Meta model, we can define 
transitions from real activity to proxy activity and vice versa. However, there is no output/input and participant attached to proxy activity, as proxy activities are not supposed to produce or consume output/input. Similarly proxy activities are not meant to be executed by local role. Also, information regarding identification of external organization, the communication protocol be used, the WfMS it has and etc., should be known to the entities of external organizations.

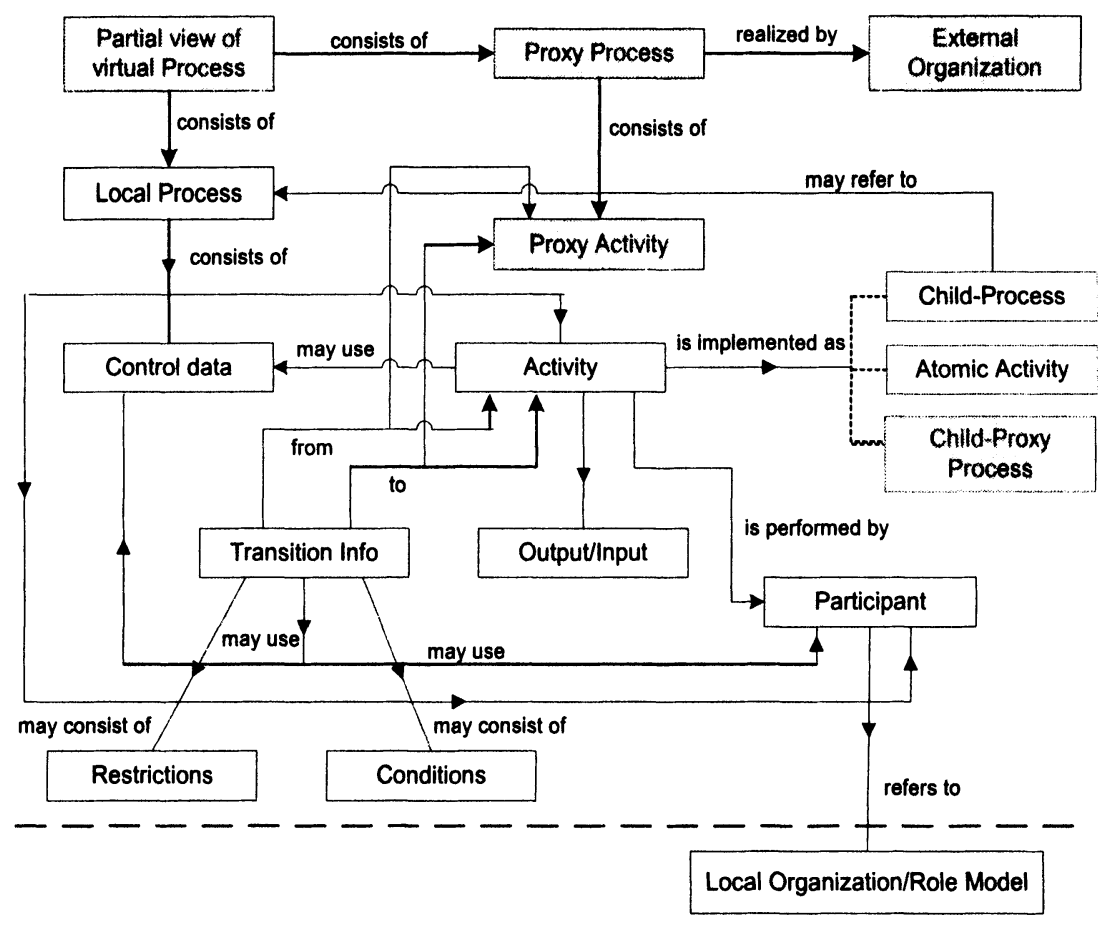

Figure 1 - Meta Model of top-level entities of Partial view of Virtual Process

It should be noted that "proxy activity" and the activity whose type is "proxy process" are two different things. If the type of the activity is "Proxy Process" it means that the activity will act as sub-process which will be realized and executed on the workflow management system of some external organization. This is to support sub-contracting as in [15].

All the other notions in figure 1 are standard notions of WFMC [4]. In short, with the help of this Meta model we can draw a network of processes and activities which may consist of normal processes or proxy processes. This network is the part of virtual process an organization can see or control autonomously. We name it 'partial view' of virtual process, as this is the view of virtual process seen by the organization. This Meta model supports all the models specified in WFMC "interoperability models" [6]. 


\section{BUILDING OF PARTIAL VIEWS OF A VIRTUAL PROCESS}

\subsection{An illustrative example}

The example in figure 2 will be used to illustrate the application of the above approach and the Meta model. This example is inspired by one of the scenarios given in [5], named "Acute Forensic Examination" (AFE). In figure 2, the local workflow processes of the participating organizations - Community Pediatrics, the Police, Examination Room Provider and Post AFE Care Provider - are modeled by using WFMC Meta model [4]. An oval shows one activity and the arrow symbolizes a transition along with the condition (the outputs of the activity are not shown here.) The splitting and joining of the activities are according to WFMC [4]. For the sake of better clarity in discussion, unnecessary activities, details and issues have been omitted.
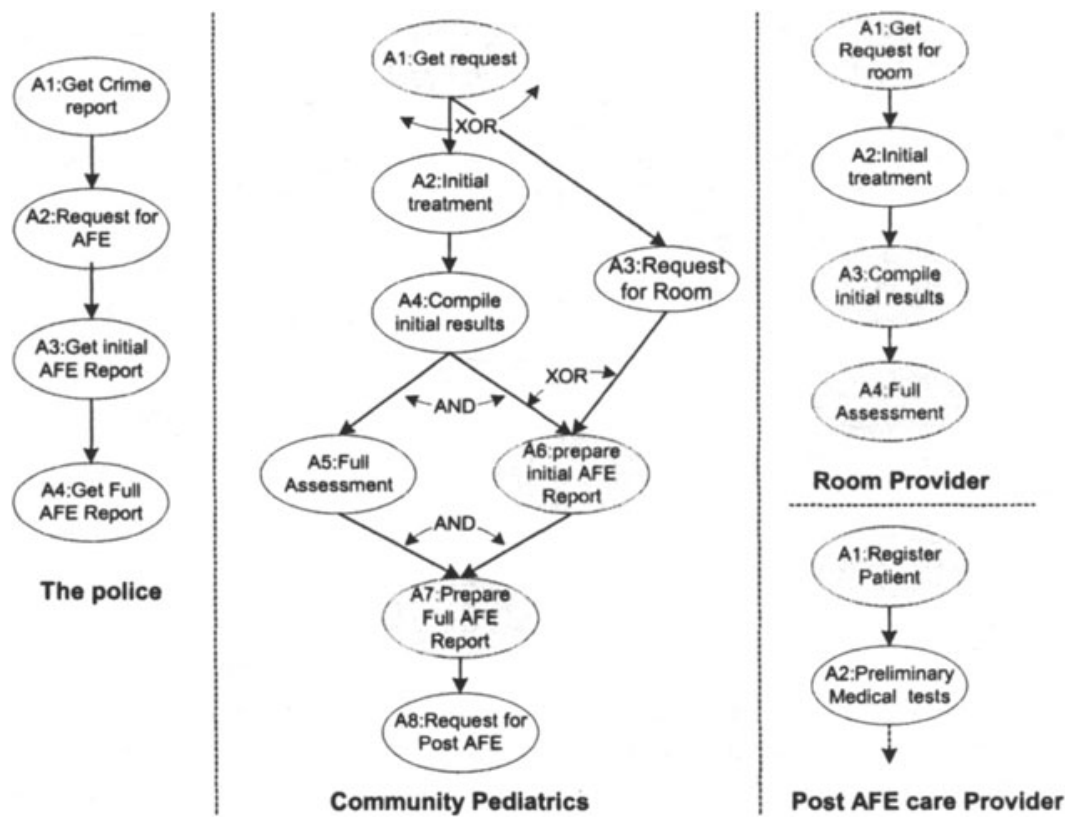

Figure 2 - Four Local Processes of Participating organizations

All the workflow processes shown in the figure 2 are self-explanatory. The only point to be noted is that the interactions between organization are manual e.g. forwarding of request of AFE to Community Pediatrics by the police. Again only the relevant parts of process are shown in the figure.

\subsection{Partial views of the Virtual Processes}

Suppose the four autonomous organizations now wish to establish a virtual enterprise as quickly as possible with least changes to be made in their local 
workflow processes created previously as shown in figure 2 . This can be done in two main steps. Firstly, every organization exposes some part of the local workflow process to the designated external organization. This is achieved by the proxy process and its proxy activities (figure 3 ). A proxy process is created for one of the following purposes: the local workflow wants to expose some of his activities or the local workflow wants to access activities of other organizations.

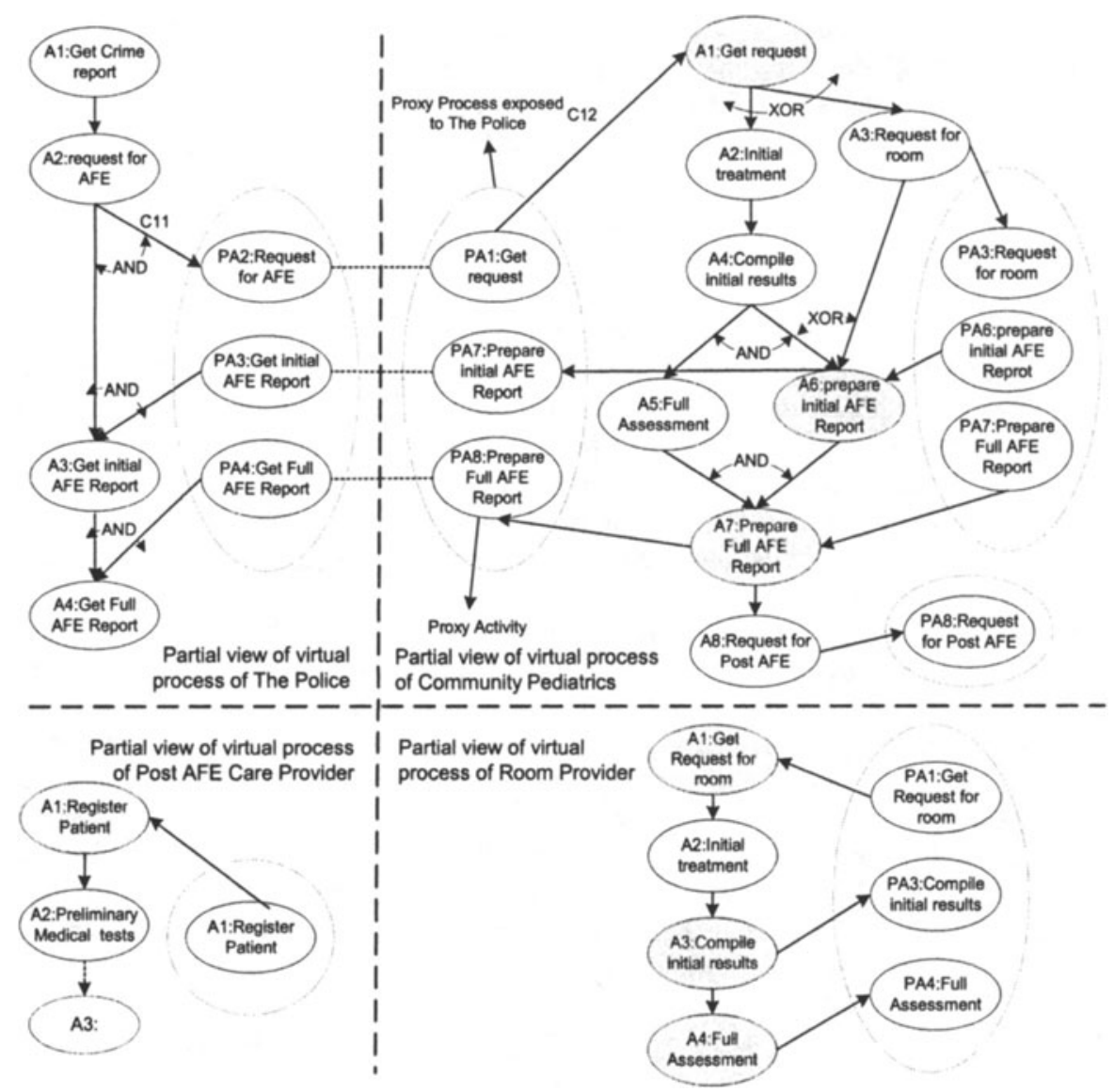

Figure 3 - Four partial views of virtual processes of participating organizations

Just to explain, we focus on the police and community pediatrics and their interactions. Based on above rules, the police create one proxy process to access community pediatrics' process. Similarly Community Pediatrics creates three proxy processes: one to expose its activities to the police and two to access external processes of room provider and AFE Post Care Provider (figure 3). The proxy process meant for the police has three proxy activities. These activities act as proxies of the activities A1, A7 and A8 (activities to be accessed by the police). For instance, activity PA1 is a proxy of activity A1. This proxy process is created and configured for accessed only by one organization: the police. If the same activity, say A7 of community pediatrics, is supposed to access activity of another external 
organization, say room provider, then a new process activity as part of another proxy process will be created for that organization. The transitions between proxy and real activities along with the conditions are defined as part of this step. Likewise, all the organizations create necessary proxy processes and its activities and configure them for accessed by the respective external organizations.

The second step is to bind proxy activities of one partial view with the counter parts of other organizations. For example, the police bind PA2 of its partial view with PA1 of community pediatrics. Please note that the workflow builder at the police sees only the exposed proxy process and its three proxy activities of the community pediatrics; all the other internal details of workflow of the latter is hidden to the Police. After this step, the partial views of the all organizations are shown in Fig. 3.

\subsection{Distributed control over virtual process}

The transition from a real activity to a proxy activity or a proxy activity to a real activity is same as the transition between real activities. But the proxy activity itself does not do anything except two: communication and distribution of control. When the transition from a local activity to a proxy activity occurs, the proxy activity sends a message to its bound proxy activity on the other WFMS. This message contains the output of the real activity which is an input of the next real activity on the receiving WfMS. This message also gives the control of the thread of activities to the workflow process on the receiving WfMS.

Let us consider the transitions $\mathrm{A} 2 \rightarrow \mathrm{PA} 2 \rightarrow \mathrm{PA} 1 \rightarrow \mathrm{A} 1$ which are shown in the figure 3. If we remove the proxy activities, the transition becomes $\mathrm{A} 2 \rightarrow \mathrm{Al}$ which is the transition of real activities belonging to two different WfMSs. For a successful transition between the real activities, both the conditions $\mathrm{C} 11$ and $\mathrm{C} 12$ have to be satisfied. As it is clear from the figure 3 , the conditions are distributed and controlled independently by the two participating organizations. Hence from the perspective of the whole virtual process, the control is distributed. In such transitions, the WfMSs involved could be heterogeneous; hence there is also a need to resolve their differences in data format and communication protocol, which is typically performed as part of the Proxy activity.

\section{VIRTUAL PROCESS ENACTMENT FRAMEWORK}

\subsection{Architecture}

The 'Virtual Process Enactment Framework' is a set of WfMSs that are collaborating in a very loosely coupled way. These WfMSs are likely to be heterogeneous. The system architecture shown in figure 4 represents major components that almost every workflow management system has except one Request Handler. The request handler facilitates participating organizations in building, executing and monitoring of virtual processes. At the build time of the virtual process, the process builder requests the request handler of an external WFMS for access to the exposed proxy processes and then binds its proxy processes with them. During the execution and monitoring of a virtual process, the workflow engine sends XML messages directly to request handler of others WfMS. For 
interoperability between WfMSs, we considered three available workflow interfaces: interface 4 of WFMC [6], JFlow [7] and SWAP [8]. We followed the standard specified by interface 4 WFMC. In spite of closest match, we had to introduce new messages because of the requirements of our approach. These messages are mainly for viewing of exposed processes, binding of processes and activity level interactions. We believe that most of the existing workflow management systems can adopt our solution, including those used in healthcare industries.

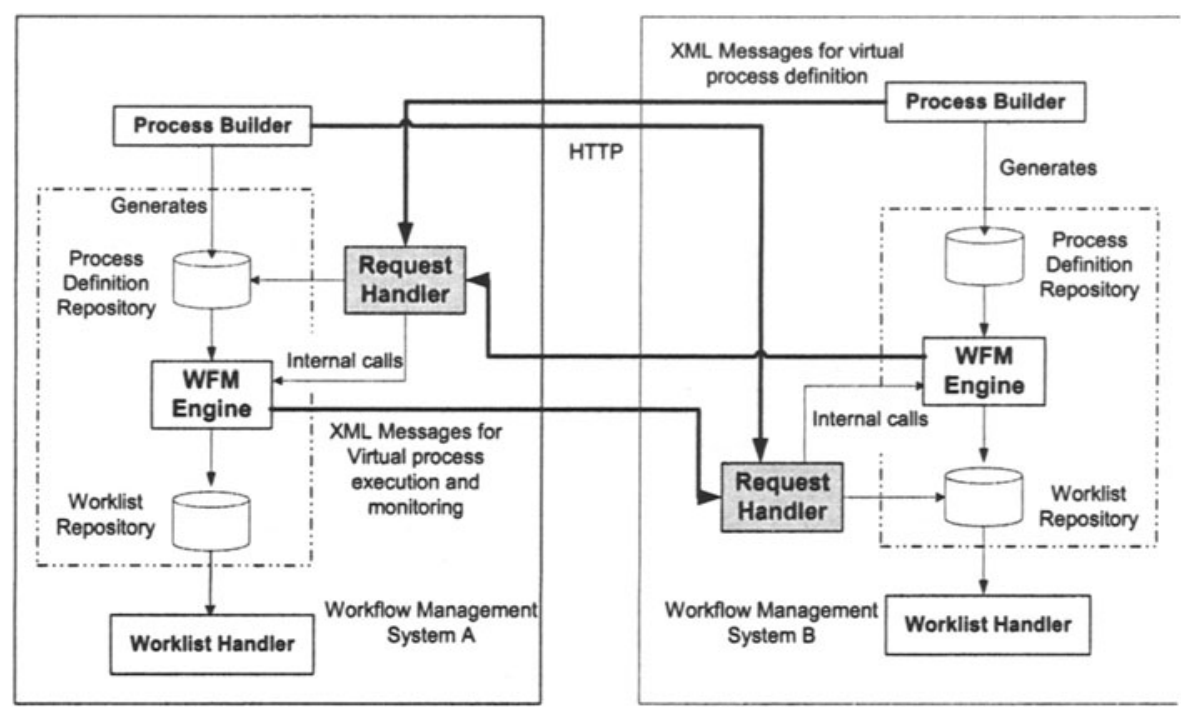

Figure 4 - Abstract Architecture of Virtual Process Enactment Framework

\subsection{Meta model for Virtual Process enactment}

Each partial view of virtual process is enacted independently and separately in a WfMS. During the enactment, the WfMS interacts with other WfMSs according to the definitions of partial view of virtual process to achieve the enactment of the whole virtual process. Like local process, a virtual process may have more than one case/instance. As the definition of a virtual process is a set of definitions of local processes, a virtual process instance is therefore a set of local process instances. Based on our model of partial view of a virtual process, we propose a Meta model of top level entities of an enactment service, as shown in figure 5. One of the important requirements here is to track which local process instance should talk to which local process instance on the other WfMS. This requirement is further complicated when the interaction occurs at the level of activities. It is therefore necessary to model the entities of the enactment service explicitly. 


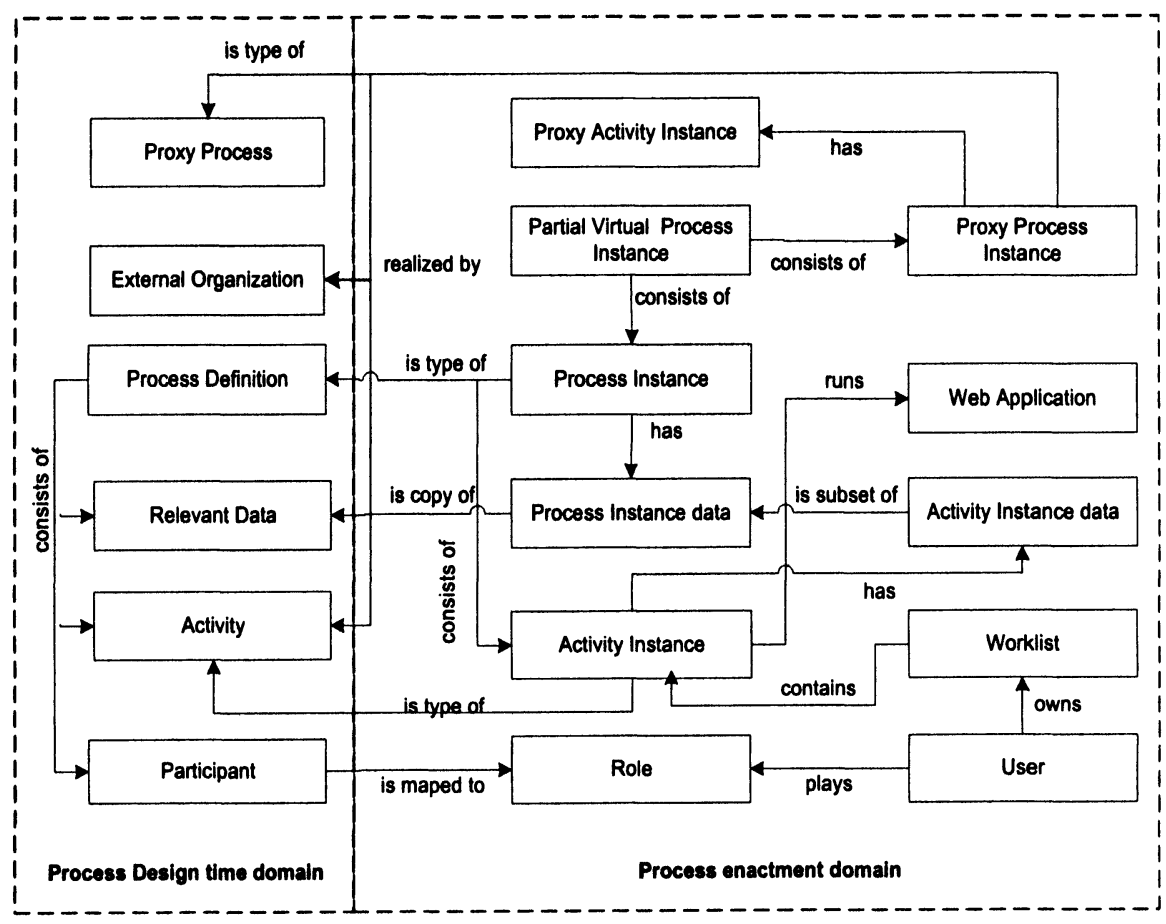

Figure 5 - Meta Model of top level entities of Virtual Process Enactment

Framework

\section{EVALUATION}

The key difference between eCommerce Virtual enterprises (or B2B, B2C, manufacturing and etc.) and Virtual Health enterprise is in their sensitivity of exposure of internal processes. In eCommerce Virtual enterprises, there is always an element of competition or secrecy among the participating organizations. The process (the way things are done) should not be exposed (e.g. it could be analyzed to reveal efficiency secret or weaknesses in responding to markets). But In Virtual Health enterprise it is not always that sensitive to expose process (of course, data and information may still be hidden). Our approach takes advantage of this liberty of Health Care Sector.

There are several advantages of our approach, which we believe other related works (See section 6) do not possess. Our partial views approach allows existing workflows to be quickly integrated into virtual process. And it could be done with minimal changes to their existing workflows. The Meta model of our partial view is not proprietary but based on international standard of process definition specified by WFMC [4]. Most importantly, the resulting virtual enterprise processes are dynamic and elastic. By dynamicity we mean the relationships of participating organization can be changed quickly. And by elasticity we mean a virtual enterprise process is extensible. Let us consider that in the context of the abovementioned example, a medical insurance company of the patient wants to be part of this virtual enterprise and to link up its payment workflow process with Post AFE Care Provider. For this, 
what it needs to do is to define its own view of virtual process and links it with the corresponding partial view of virtual process of the Post AFE Care Provider. Clearly, this integrating process is fast and yet transparent to the remaining organizations of the virtual enterprise - the Community Pediatrics, the Police and the Room Provider.

A main challenge of our solution (as well as many inter-organizational workflow solutions) is its difficulty in ensuring correctness of virtual process. A virtual process constituted by a set of individually validated local workflows may be incorrect (such as having deadlocks and looping). Unfortunately existing local process building and analysis tools are still inadequate in ensuring the correctness of the overall virtual process.

\section{RELATED WORK}

We can categorize approaches of virtual process (cross-organizational process) into three categories: split and deploy, composition and black box. In Split and deploy a whole process is designed and then it is split into several sub-processes for deployment in different organizations. This approach is typified by METEOR project [9] and "Public to private" [10]. In the approach of "composition" a whole process specification is obtained from several segments that may be contributed by different organizations. Then the process is built and deployed. WISE [11] and CMI [12] take this approach. A good representative work of this approach is process fragments [13]. The black box approach introduced by CrossFlow [14] lets the participating organizations to specify their collaboration in a central electronic contract. Based on this contract, services are configured and outsourced among participating organizations of virtual enterprise.

Our solution falls into the category of "composition". But the key difference between other solutions and ours is that, in our case, the activities of local processes are exposed directly through proxies. METEOR and "Public to private" use an opposite mechanism in their approaches. And comparing with CrossFlow, we do not have a central contract for establishing the virtual process/virtual enterprise. Our interaction of processes is peer-to-peer.

In the CMI [12], a project at MCC, inter-organizational workflow was achieved by using a Collaboration Management Model (CMM) sitting on top of other workflow models to map methods and tools for defining services provided by different companies. The basic idea of CMI is the integration of processes via interenterprise services. CMI does not aim at designing a new workflow management system. They assume that processes are enacted by a commercial WfMS instead. In WISE [11], a web based e-commerce platform project, process designers can post their design segments into a common World Wide Web (WWW) based catalog repository. A virtual process specification can be constructed by using the segments retrieved from the catalog. This specification can then be compiled and the resulting processes be enacted by the WISE engine. This paper does not however present a concrete process model for the specification of cooperating processes, and does not show how the interaction is achieved. 


\section{CONCLUSION}

This paper proposes a method and framework for the development of virtual healthcare enterprises. The method exploits the fact that internal processes of healthcare organization can be exposed. We demonstrate the application of this method to the design of virtual processes through a real world example. The architecture of the proposed virtual process enactment framework being implemented is also presented in the paper. This architecture is extended from our previous design for intra-organizational workflow processes. The Meta model of top-level entities of the enactment framework is also presented. Our work demonstrates the feasibility of adapting existing WfMSs for intra-organizations to inter-organizations.

\section{REFERENCES}

1. Alexander Horsch and Thomas Balbach, Telemedical Information Systems IEEE TRANSACTIONS ON INFORMATION TECHNOLOGY IN BIOMEDICINE, VOL. 3, NO. 3, SEPTEMBER 1999 (good reference.pdf)

2. Deloitte \& Touche, The emerging European Health Telematics Industry, February 2000. Reference C13.25533 (ref: /medical/HIST-rep.pdf)

3. E. Maij 1 , V.E. van Reijswoud 2 , P.J. Toussaint 3, E.H. Harms 4 , J.H.M. Zwetsloot-Schonk 5, A Process View of Medical Practice by modeling Communicative Acts, Methods of Information in Medicine. Vol. 39, No.1, pp 56-62, 2000.

4. WFMC Interface 1, Process Definition Interchange, http://www.wfmc.org/standards/docs.htm

5. ERDIP, Cross Boundary Information Flows http://www.nhsia.nhs.uk/erdip/pages/publications/DemonCrossBoundaryInformationFlows.pdf

6. WFMC Interface4, Interoperability, http://www.wfmc.org/standards/docs.htm

7. OMG jFlow Submission, ftp://ftp.omg.org/pub/docs/bom/98-06-07.pdf

8. Simple Workflow Access (SWAP), http://www.ics.uci.edu/ ietfswap/index.html

9. W.M.P. van der Aalst, K. Anyanwu, Inheritance of Interorganizational Workflows to Enable Businessto-Business E-commerce, Proceedings of the Second International Conference on Telecommunications and Electronic Commerce (ICTEC'99), pages 141-157, October 1999

10. W.M.P. van der Aalst, M. Weske, The P2P Approach to Interorganizational Workflows, Proceedings of the 13th International Conference on Advanced Information Systems Engineering (CAiSE'01), pages 140-156 Springer-Verlag, Berlin, 2001

11. A. Lazcano, G. Alonso, H. Schuldt, and C. Schuler. The WISE approach to electronic commerce. Int. Journal of Computer Systems Science \& Engineering, 15(5), September 2000.

12. D. Georgakopoulos, H. Shuster, A. Cichocki, and D. Baker. MANAGING PROCESS AND SERVICE FUSION IN VIRTUAL ENTERPRISES, Information Systems Vol. 26, No. 6, pp. 429 \{456, 1999

13. Lindert, Frank and Deiters, Wolfgang, Modelling inter-organizational processes with process model fragments, Proceedings of the Workshop Informatik '99, 1999

14. Paul Grefen, Karl Aberer, Yigal Hoffner \& Heiko Ludwig, CrossFlow: Cross-Organizational Workflow Management in Dynamic Virtual Enterprises

15. W.M.P. van der Aalst, Interorganizational workflow: An approach based on message sequence charts and Petri nets, Systems Analysis - Modelling - Simulation, Vol. 34, No. 3, pages 335-367. 1999 